\title{
Possibilities of Minimally Invasive Treatment of Hip Subluxation in Children with Cerebral Palsy
}

\section{Peter Bernius}

Chief Physician, Schön Klinik Munich Harlaching, Germany

\section{Abstract}

Introduction: Hip subluxation is one of the main problems in children with cerebral palsy (CP) after the equines foot. Without treatment, we see a deterioration of around $7 \%$ per year. In addition to the delayed verticalization, the main cause is the pathological muscle tone of the muscles surrounding the hip. Children with restricted ability to walk and stand independently are most affected, which is assessed using the classification system for gross motor function (GMFCS). It is a common therapeutic measure to treat muscle imbalance by releasing the shortened soft tissue in early childhood. Here we prefer percutaneous myofasciotomy to lengthen short muscles. The Iliopsoas muscle cannot be lengthened percutaneously.

Since muscle lengthening is not sufficient for older children with hips at risk, we use additional guided growth as a minimally invasive method to prevent further hip subluxation. In this regard, two other studies already showed a positive effect.

Methods: This was a prospective follow-up study from 2010 to 2016 of 52 children aged 2.8 to 6.7 years ( 29 boys and 23 girls) who were to be treated surgically with a percutaneous muscle fascia release due to structural muscle shortening. In the preoperative examination, an X-ray image revealed a Reimer migration index (RMI) of over $25 \%$. All children were classified according to their functional possibilities (GMFCS II 3 children, III 14 children, IV 18 children, V 17 children) and the mobility of both legs (ROM) was measured. The second group of 12 children over the age of 6 ( 6.1 to 12.7 years) ( 7 boys, 5 girls) underwent femoral head epiphysiodesis in addition to the release of the fascia between 2016 and 2018. The group was also examined according to the same criteria (III 2 children, IV 5 children, V 5 children). We followed these children postoperatively and reevaluated radiological and clinical findings.

Results: For 99 observed hips of 52 children with CP GMFCS 3-5 younger than 6 years of age (average 4.2 years), we found an improvement in hip subluxation for $33 \%$, deterioration for $5 \%$ and stabilization for $62 \%$.

For our patients older than 6 years, we combined the percutaneous myofasciotomy with a guided growth of the femoral head by percutaneous screw. For 16 hips of 12 children we found a mean improvement in the migration index of 5\%, and an improvement in the femoral neck shaft angle of $9^{\circ}$ within 16 months.

Discussion: The minimally invasive release of muscle fascia appears to be as effective as the open release, as it is done with hip screening programs in many countries. The recovery of children who receive minimally invasive treatment is faster, however. Less surgical soft tissue damage, faster mobilization and fewer restrictions than varus osteotomy are strong arguments to consider guided growth as a treatment option for Coxa valga in spastic hip subluxation in non-ambulant children with CP.

Conclusion: Further studies have to prove the effectiveness of this method in the long term.

\section{Introduction}

Hip subluxations often occur in children with $\mathrm{CP}$, especially in children who are unable to walk. Population-based studies have shown that the rate of hip subluxation increases significantly, depending on the child's reduced functional level, from $0-5 \%$ in level I of the Gross Motor Function Classification System (GMFCS) [1] to 70-90\% in level $\mathrm{V}$ [2-5]. The greatest risk of hip subluxation is between the ages of 4 and 12 years [6]. If the hip remains untreated, the hip lateralization progresses at an average RMI $+7 \%$ per year depending on age and walking ability [7]. When a complete displacement occurs, hip pain and profound problems with the walking ability, sitting balance, and genital care may occur $[8,9]$. Therefore, screening programs for the early detection and treatment of hip subluxations were developed $[2,10,11]$.

Soft tissue procedures such as adductor and psoas releases have been recommended as prophylaxis to prevent hip displacement. Although such procedures had a good effect in studies with a mean

\section{Publication History:}

Received: April 08, 2020

Accepted: June 30, 2020

Published: July 01, 2020

\section{Keywords:}

Cerebral palsy, hip subluxation, preventive surgery, myofasciotomy, minimal invasive surgery, orthopedic management, percutaneous screw epiphyseodesis follow-up time of 3-4 years in $70-80 \%$ of the hips [12-14], the rate in other studies with a longer follow-up period was smaller and the recurrence rate above 50\% [15,16]. It has not been clarified when and why the operation fails. It is therefore unclear whether fails are caused by a poor primary correction of subluxation or by a later relapse after a satisfactory primary correction. Further research needs to be done.

After the soft tissue procedures, the patient usually was immobilized in abduction splints for at least 6 weeks. The physiotherapy started

*Corresponding Author: Dr. Peter Bernius, Chief Physician, Schön Klinik Munich Harlaching, Germany; E-mail: pbernius@schoen-klinik.de

Citation: Bernius P (2020) Possibilities of Minimally Invasive Treatment of Hip Subluxation in Children with Cerebral Palsy. Int J Pediatr Neonat Care 6: 167. doi: https://doi.org/10.15344/2455-2364/2020/167

Copyright: (C) 2020 Bernius. This is an open-access article distributed under the terms of the Creative Commons Attribution License, which permits unrestricted use, distribution, and reproduction in any medium, provided the original author and source are credited. 
Citation: Bernius P (2020) Possibilities of Minimally Invasive Treatment of Hip Subluxation in Children with Cerebral Palsy. Int J Pediatr Neonat Care 6: 167. doi: https://doi.org/10.15344/2455-2364/2020/167

Page 2 of 6

afterward. It wasn't analyzed whether this immobilization also had a bad impact on the results.

In the Terjesen [5] study, surgical treatment was recommended for children with a migration index (MP) of over 33\%. According to the guidelines of the Norwegian working group, soft tissue releases should be used for mild and moderate subluxation, while bony procedures should also be carried out for children with severe subluxation (MP $\geq 50 \%$ ).

In the Hägglung [3] study, the risk of subluxation ( $\mathrm{MP}>40 \%)$ was directly related to the degree of gross motor function, classified according to the GMFCS, reaching from $0 \%$ for children in GMFCS level I to $64 \%$ in GMFCS level V. Since lack of movement will probably have a negative influence on muscle mass and elasticity, as emphasized by Silvferskjöld [17], postoperative immobilization and fixation, as little invasive and less painful surgical procedures as possible and early functional rehabilitation treatment were sought. Percutaneous myofasciotomy was chosen as an alternative to the open soft tissue release, and percutaneous femoral neck screw epiphysiodesis according to Lee [18] was chosen as an alternative to derotation-varus-osteotomy of the proximal femur in children over the age of 6 .

The rehabilitation program included the movement of all muscles and joints up to the pain limit from the first postoperative day, full load bearing when standing, starting with assisted movements and increasing, as far as possible, to independent movements, lymphatic drainage from the first day against lymphatic congestion and hematomas.

We hypothesized that this therapy regime can achieve at least the same result as open muscle and tendon release described in earlier studies.
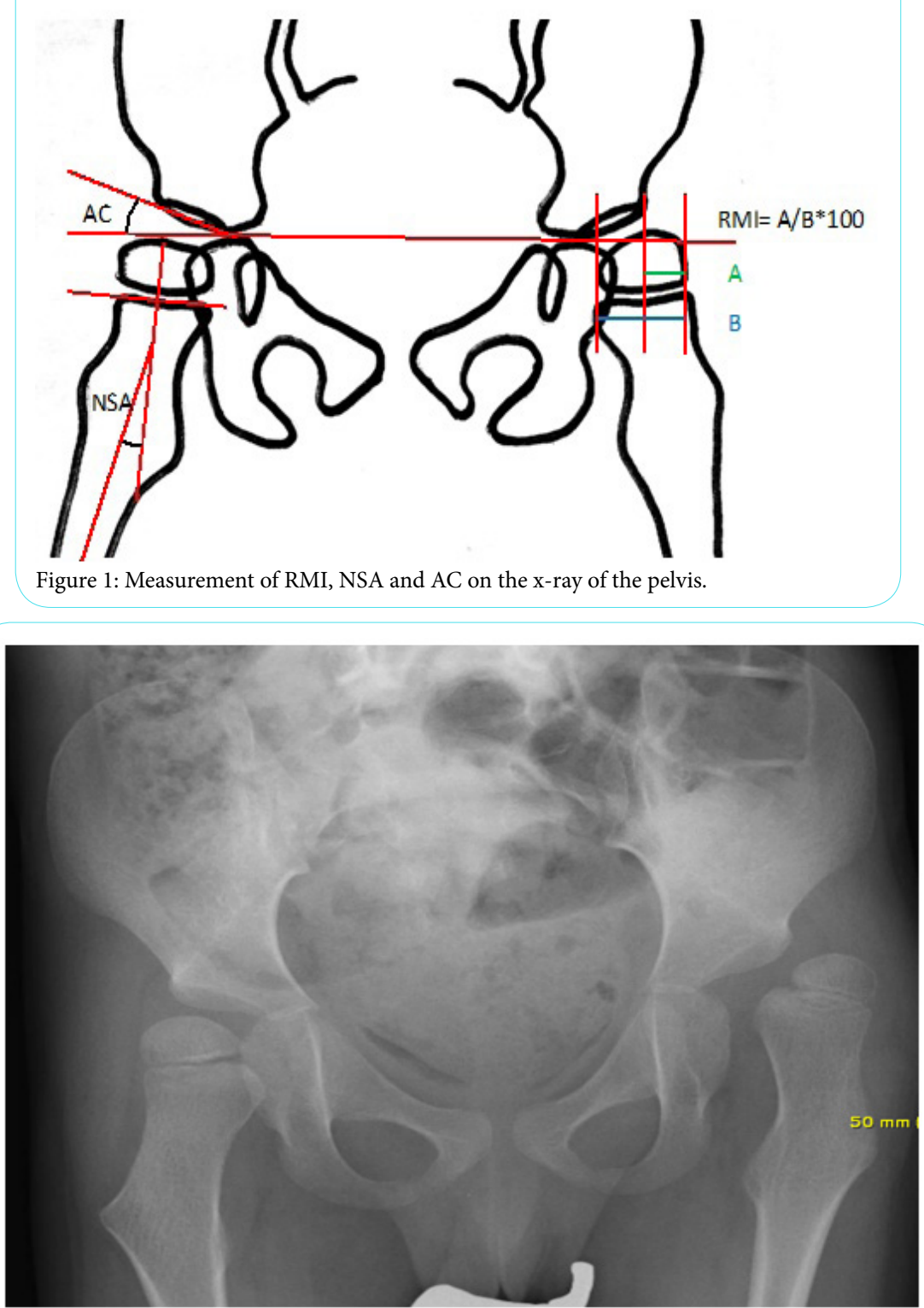

Figure 2: Boy GMFCS 5, 7 years old with complete dislocation of the left hip left and subluxation on the right. 
Citation: Bernius P (2020) Possibilities of Minimally Invasive Treatment of Hip Subluxation in Children with Cerebral Palsy. Int J Pediatr Neonat Care 6: 167. doi: https://doi.org/10.15344/2455-2364/2020/167

Page 3 of 6

\section{Patients and Methods}

The study program was started in our hospital in 2010. All children with $\mathrm{CP}$ who were presented in the hospital or in our external outpatient clinic were indicated for soft tissue release because of severe muscle contractures and showed a RMI above $25 \%$ on the X-ray were included (Figure 1). The current investigation includes the results for patients up to operations in early 2016, allowing a follow-up period of at least 4 years. The diagnosis and type of $\mathrm{CP}$ were made according to the SCPE 2000 guidelines. The study program includes systematic clinical and radiological follow-up examinations. The movement deflection was measured clinically and classified according to GMFCS and radiologically the Reimer migration index (RMI), the acetabular angle (AC) and the projected femoral neck shaft angle (NSA) were measured.

The present study included 52 children with 99 critical hips who had undergone percutaneous soft tissue release. The mean age at surgery was 4.8 (2.8-6.7) years. 43 children had bilateral CP (14 of them diplegic) and 4 children dyskinetic CP (variable muscle tone) (Figure 2).

The percutaneous myofasciotomy took place depending on the clinical examination findings. To influence the development of the hip, muscles that have a direct influence on the movement of the hip joint were myofasciotomised. These were the adductor longus, the gracilis, the tensor fasciae latae, the gluteus, the rectus femoris, the semitendinosus, the semimembranosus and the quadriceps. As part of the operation, muscles not directly influencing the movement of the hip joints, which can have an indirect influence on muscle tone via the muscle chainswere also treated.

The adductor longus muscle was treated if the abduction was below $60^{\circ}$ with the hip and knee flexed, the gracilis if the abduction was not possible with the hip and knee extended up to at least $40^{\circ}$. In the absence of hip extension to $0^{\circ}$ with the Thomas grip on the opposite side, the tensor fasciae latae and, if that alone was insufficient, the rectus femoris were released. In case of spontaneous external rotation less than $20^{\circ}$ in hip extension the gluteus medius was addressed. The popliteal angle should not be greater than $30^{\circ}$, otherwise, the semitendinosus and the semimembranosus were myofasciotomised and inoverhang the knee flexion with the hip in extension should be

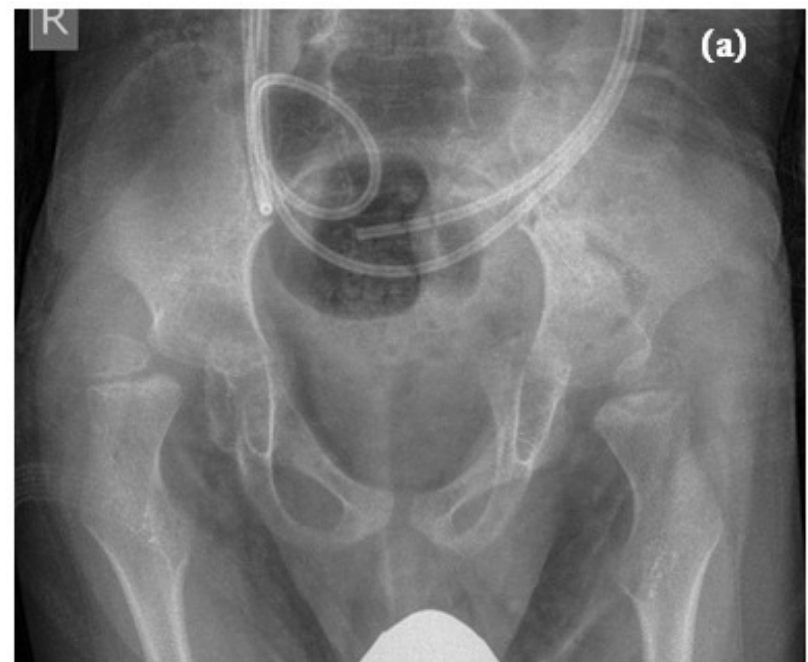

at least $70^{\circ}$. Otherwise, myofasciotomy of the quadriceps muscle was performed. All ranges of movement were checked only with slight pressure.

The rehabilitation was carried out early on with functional movement and full load, lymphatic drainage and hematoma treatment as well as splints in extension and abduction during rest.

As of April 2016, we conducted a prospective study with surgical treatment with myofasciotomy and in combination with femoral neck epiphysiodesis in children over the age of 6 if they were presented in the hospital or in our external outpatient clinic, were indicated for soft tissue release because of severe muscle contractures showing an MP of over $25 \%$ on the X-ray of the pelvis. So far, 16 hips of 12 children over the age of 6 have been included. The mean age at the operation was 7.9 (6.1-12.7) years. All children had a bilateral CP (5 of them diplegic). In total, we have now performed this treatment in over 160 hips. The range of movement was clinically measured and classified according to GMFCS and radiologically the RMI, the AC and the projected NSA were measured (Figure $3 \mathrm{a}$ and $3 \mathrm{~b}$ ).

The muscles were treated in the same way as in children up to the age of 6 years. In addition, a self-drilling and self-tapping banjo screw was inserted percutaneously in the children approximately 3 to $5 \mathrm{~cm}$ below the greater trochanter apophysis in the axis of the femoral neck through the femoral epiphyseal plate under fluoroscopic control. The placement in the cortex of the shaft was done using a Kirschner wire. The placement in the femoral neck, however, was done directly without placing the Kirschner wire (Figure $4 a, 4 b$ and $4 c$ ).

In this group, too, the rehabilitation was carried out early in terms of function, with immediately permitted movement and full load, lymphatic drainage and hematoma treatment, as well as splints in the extension and abduction during rest. Special protection was not required.

The Ethics Committee of the Schön Clinic Munich Harlaching approved ethical permission. No additional examinations or therapies were performed in comparison to our regular surveillance. Our treatment regime in children older than 6 years with hips at risk had guided growth treatment instead of varisation osteotomy.

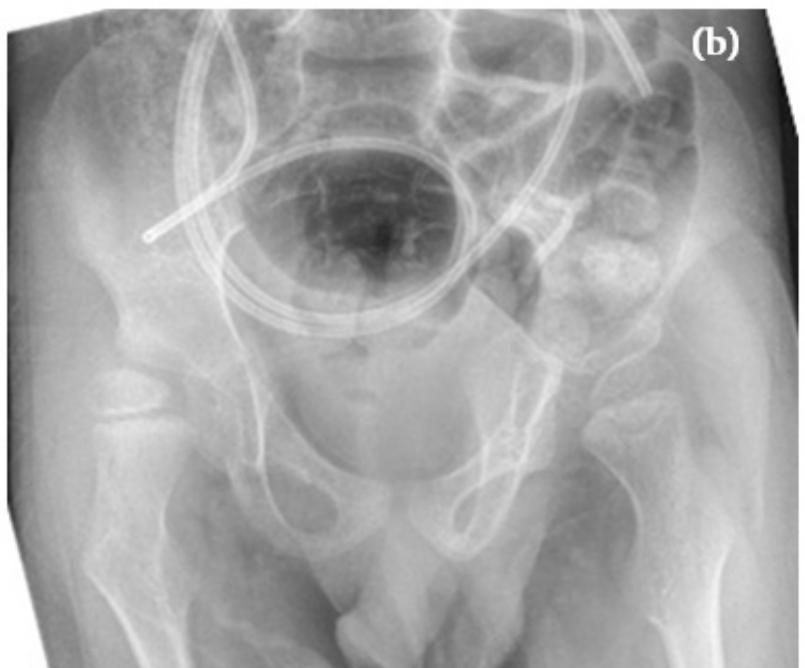

Figure 3: (a) Boy GMFCS 5, 5-years-old, pre OP. (b) Boy GMFCS 5, 14 months postmyofasciotomy.

Int J Pediatr Neonat Care

ISSN: 2455-2364
IJPNC, an open access journal Volume 6. 2020. 167 
Citation: Bernius P (2020) Possibilities of Minimally Invasive Treatment of Hip Subluxation in Children with Cerebral Palsy. Int J Pediatr Neonat Care 6: 167. doi: https://doi.org/10.15344/2455-2364/2020/167
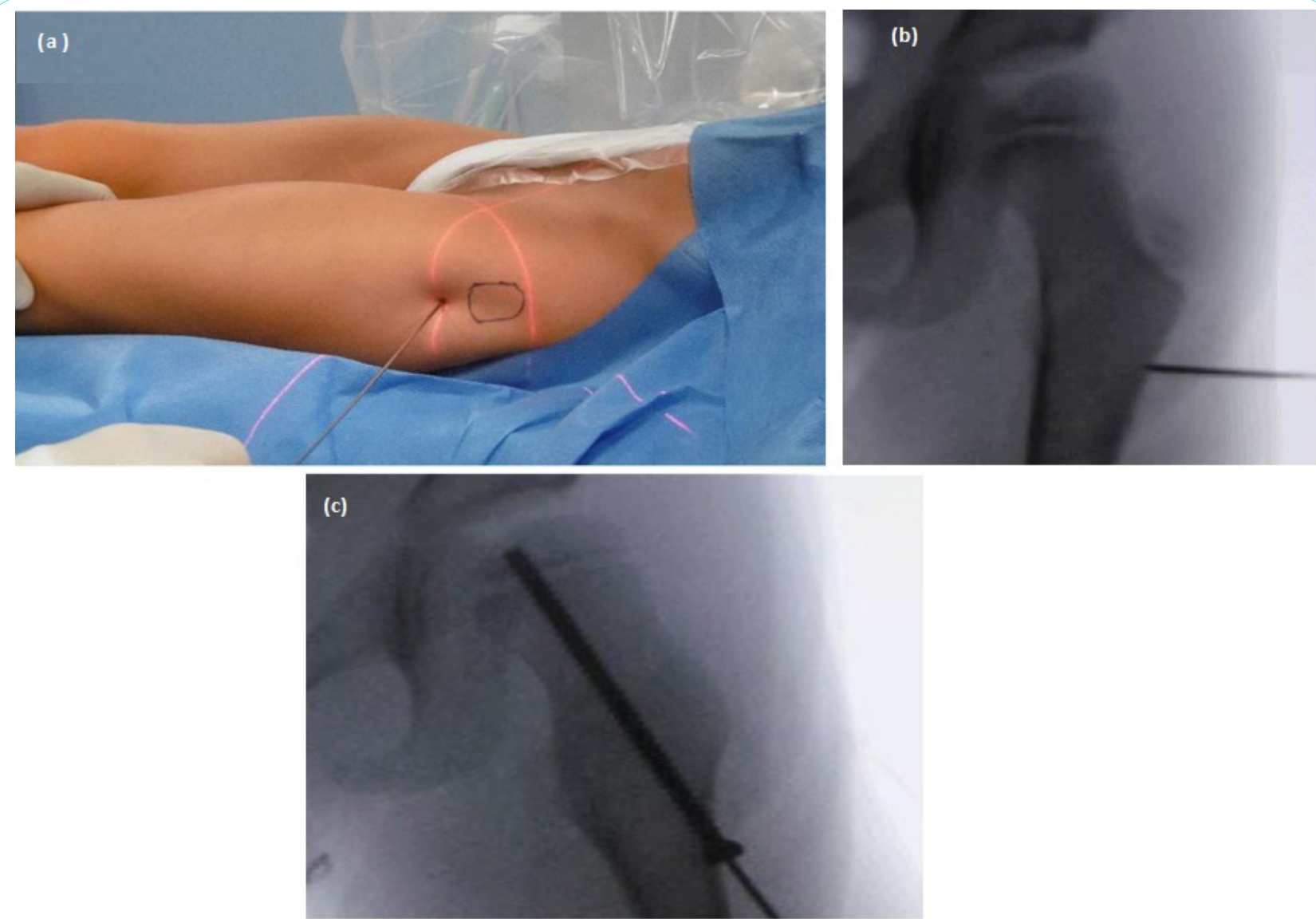

Figure 4: (a) Intraoperative situation, marking the entry point for the screw with the help of a wire. (b) Intraoperative fluoroscopy: wire approx. 2-3 $\mathrm{cm}$ below the greater trochanter. (c) Intraoperative fluoroscopic image: screw aligned in the axis of the femoral neck and good grip of the epiphyseal gap just slightly medial to the middle of the physis.
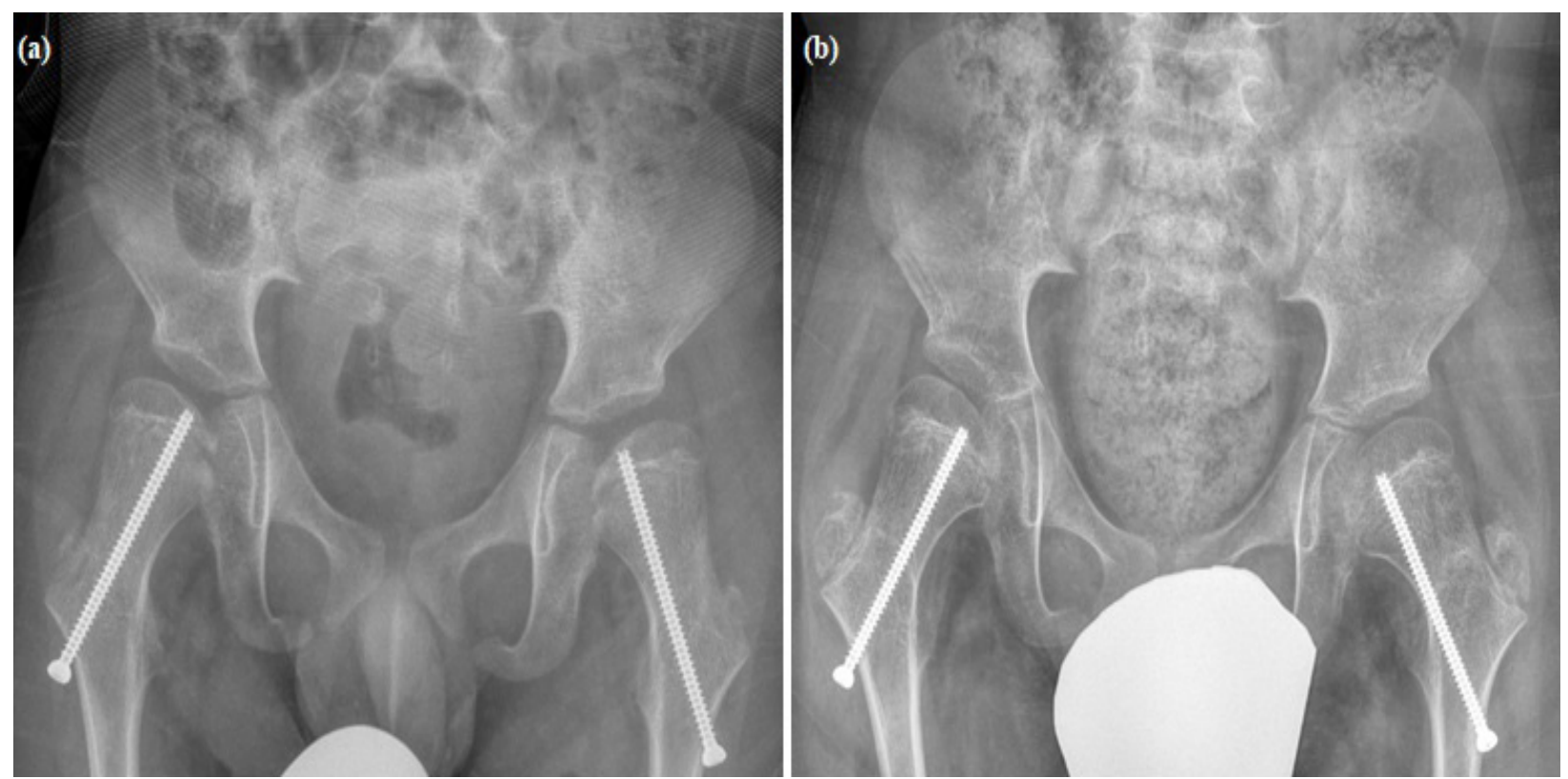

Figure 5: (a) Boy GMFCS 4, 8 years old, directly after the operation. Screw location relative to middle of epiphysis and trochanter not important. Enough windings of screw need to fix epiphysis. (b) Boy GMFCS 4, 19 months after surgery. Clearly recognizable varus tilt of the femoral neck and epiphyseal plate. Screws only briefly hold epiphyseal joints. 
Citation: Bernius P (2020) Possibilities of Minimally Invasive Treatment of Hip Subluxation in Children with Cerebral Palsy. Int J Pediatr Neonat Care 6: 167. doi: https://doi.org/10.15344/2455-2364/2020/167

Page 5 of 6

\section{Results}

In the group of children under 6 years with myofasciotomy, we found improvement in RMI of $33 \%$, deterioration of $5 \%$ and stabilization of $62 \%$. We divided into 3 groups with initial RMI $25-39 \%$, RMI 40 $49 \%$ and RMI $50 \%$. The RMI improved from an average of $31 \%$ to $30 \%$ in the group with moderate subluxation, from $43 \%$ to $36 \%$ in the group with strong subluxation and from $69 \%$ to $62 \%$ in the group with extreme subluxation.

The AC improved in the group with moderate subluxation from an average of $23^{\circ}$ to $19^{\circ}$, in the group with strong subluxation from $28^{\circ}$ to $24^{\circ}$ and in the group with extreme subluxation from $40^{\circ}$ to $29^{\circ}$.

In the follow-up examination depending on the GMFCS, the AC improved in patients in level 3 from $26^{\circ}$ to $22^{\circ}$, in level 4 from $26^{\circ}$ to $21^{\circ}$ and in level 5 from $40^{\circ}$ to $26^{\circ}$. Depending on age, the RMI improved by an average of $-4.5 \%$ per year for 2 to 3 -year-old children, by $-5.7 \%$ for 3 to 4 -year-olds and by $-3.7 \%$ for 4 to 5 -year-olds, for the $5-6$-year-olds by $-2.2 \%$ and for the $6-7$-year-olds by $-5.6 \%$ (Figure 5 a and $5 b)$.

We found no differences in the development of RMI according to the different GMFCS levels. The children with level 2 showed an average improvement of $-6.0 \%(+0.6 \%$ to $-11 \%)$, the group level 3 of $-4.4 \%(+2.4 \%$ to $-13.3 \%)$, at level 4 of $-5.0 \%(+13 \%$ to $-57.6 \%)$ and finally level 5 of $-2.9 \%$ per year from the baseline. Over the entire examination period, the hips improved by $10 \%$ or more in 33 hips (33\%), the RMI did not change for 61 hips (deviation from the baseline values less than $10 \%$ and the RMI deteriorated by $10 \%$ in 5 hips) and more.

For our patients older than 6 years who were treated with myofasciotomy and femoral neck epiphysiodesis, we found a mean improvement in RMI of $5 \%$ for 16 hips and a $9^{\circ}$ NSA improvement at 16 months follow up. More precise and somewhat longer-term results are currently being evaluated and will be published in a next article.

\section{Discussion}

Hip instability is one of the most common orthopedic problems in CP and can lead to subluxation or displacement before skeletal maturity begins. Therefore, orthopedic management should be carried out according to the age of the patient and the severity of the pathology as well as the functional and mobilizing capacity. Most clinicians prefer surgical treatment as subluxation progresses. Unlike in other published studies, we prefer early percutaneous release of the muscle fascia instead of open tendon releases in children with CP under the age of 6 and additional early guided growth when the femoral neck is steep rather than a varus osteotomy in children over the age of 6 . Both treatments allow an immediate full load and movement exercises up to the pain limit. An immobilization as with tendon releases and varus osteotomies is not necessary. The disadvantages of immobilization, pain-related passivity and relief with muscle atrophy and fibrosis of soft tissues are, therefore, less expected. These minimally invasive, gentler interventions also can stop the subluxation for most and even converted into a slight improvement for some cases. According to Terjesen [7] a untreated hip at risk develops a worsening of + 7\% RMI per year. According to our results, the positive effect of percutaneous soft tissue release is independent of the existing MP, the GMFCS and the age in the examined age range from 2 to 6 .
The current study shows an increased MP in GMFCS IV and V at the time of surgery. All patients with very high MP were from groups GMFCS IV and V. These results are similar to those of other studies.

The classification of our patient group according to GMFCS showed that there were 3 patients in stage II, 9 patients in stage III, 19 patients in stage IV and 21 patients in group V. After the operation, there were 2 Level I patients, 4 Level II patients, 10 Level III patients, 19 Level IV patients and 15 Level V patients. Mutlu et al., [19] found a slight change in measurements in the GMFCS- level in 30 patients with $\mathrm{CP}$ through surgical treatment. The preoperative classification of the patient group according to GMFCS showed that there was 1 patient in level I, 4 patients in level II, 5 patients in level III, 9 patients in level IV and 11 patients in level V. After the operation, there were 4 level I patients, 2 level II patients, 5 level III patients, 10 level IV patients and 9 level V patients. Barakat et al. [20] used the GMFCS to evaluate the functional results of 22 patients with $\mathrm{CP}$ with whole-body involvement in which soft tissue release and bony surgery were performed due to hip subluxation or dislocation. Before the operation, 5 patients were level III, 7 level IV, 10 level V, while after the operation 9 were level III, 6 level IV and 7 level 5 (20). In the study of Root et al. [21], at the end of a mean follow-up period of 7 years, coverage of the femoral head, a painless hip and an improvement in walking ability by at least one level was achieved. In our study, the GMFCS level remained constant for 37 patients and improved for 15 . There was no decrease in GMFCS in any of the patients.

Terjesen [22] recommended a bilateral soft tissue release in ambulant and non-ambulant children with $\mathrm{CP}$ and existing hip subluxation. He recommended an early soft tissue release before $50 \%$ MP occurred.

Minimally invasive release of muscle fascia appears to be as effective as open release, as it is done with hip screening programs in many countries. The recovery of children who receive minimally invasive treatment is faster. Less surgical soft tissue damage, faster mobilization and fewer restrictions than with a varus osteotomy make guided growth a treatment option for coxa valga in the spastic hip dislocation in non-ambulant children with cerebral palsy.

\section{Conclusion}

Our initial clinical findings and the data obtained from the study led us to significantly reduce open procedures and to perform minimally invasive procedures as often as medically justifiable. Further studies have to prove the effectiveness of this methods in the long term.

\section{Competing Interests}

The author declare that there is no competing interests regarding the publication of this article.

\section{References}

1. Palisano R, Rosenbaum P, Walter S, Russell D, Wood E, et al. (1997) Development and reliability of a system to classify gross motor function in children with cerebral palsy. Dev Med Child Neurol 39: 214-223.

2. Soo B, Howard JJ, Boyd RN, Reid SM, Lanigan A, et al. (2006) Hip displacement in cerebral palsy. J Bone Joint Surg 88: 121-129.

3. Hägglund G, Lauge-Pedersen $H$, Wagner $P$ (2007) Characteristics of children with hip displacement in cerebral palsy. BMC Musculoskelet Disord 8: 101.

4. Connelly A, Flett P, Graham HK, Oates J (2009) Hip surveillance in Tasmanian children with cerebral palsy. J Paediatr Child Health 45: 437-443. 
Citation: Bernius P (2020) Possibilities of Minimally Invasive Treatment of Hip Subluxation in Children with Cerebral Palsy. Int J Pediatr Neonat Care 6: 167. doi: https://doi.org/10.15344/2455-2364/2020/167

5. Terjesen T (2012) The natural history of hip development in cerebral palsy. Dev Med Child Neurol 54: 951-957.

6. Flynn JM, Miller F (2002) Management of Hip Disorders in Patients With Cerebral Palsy. J Am Acad Orthop Surg 10: 198-209.

7. Terjesen $T$ (2006) Development of the hip joints in unoperated children with cerebral palsy: A radiographic study of 76 patients. Acta Orthop 77: 125-131.

8. Samilson RL, Tsou P, Aamoth G, Green W (1972) Dislocation and subluxation of the hip in cerebral palsy. J Bone Joint Surg Am 54: 863-873.

9. Letts M, Shapiro L, Mulder K, Klassen O (1984) The windblown hip syndrome in total body cerebral palsy. J Pediatr Orthop 4: 55-62.

10. Hägglund $G$, Andersson $\mathrm{S}$, Düppe $\mathrm{H}$, Lauge-Pedersen $\mathrm{H}$, Westbom $\mathrm{L}$, et al. (2005) Prevention of dislocation of the hip in children with cerebral palsy: The first ten years of a population-based prevention programme. J Bone Joint Surg Br 87: 95-101.

11. Shrader W, Wimberly L, Thompson R (2019) Hip surveillance in children with cerebral palsy. J Am Acad Orthop Surg 27: 760-768.

12. Sharrard WJW, Allen JMH, Heaney SH, Prendiville GRG (1975) Surgical prophylaxis of subluxation and dislocation of the hip in cerebral palsy. J Bone Joint $\mathrm{Br}$ 57: 160-166.

13. Kalen V, Bleck EE (1985) Prevention of spastic paralytic dislocation of the hip. Dev Med Child Neurol 27: 17-24.

14. Miller F, Dias RC, Dabney KW, Lipton GE, Triana M, et al. (1997) Soft-tissue release for spastic hip subluxation in cerebral palsy. J Pediatr Orthop 17: 571-584.

15. Turker RJ, Lee R (2000) Adductor tenotomies in children with quadriplegic cerebral palsy: Longer term follow-up. J Pediatr Orthop 20: 370-374.

16. Shore BJ, Yu X, Desai S, Selber P, Wolfe R, et al. (2012) Adductor surgery to prevent hip displacement in children with cerebral palsy: The predictive role of the Gross Motor Function Classification System. J Bone Joint Surg Am 94: 326-334.

17. Silvferskjöld (1924) Manual De ortopedia Y Tramumatologia.

18. Lee WC, Kao HK, Yang WE, Ho PC, Chang CH, et al. (2016) Guided Growth of the proximal femur for hip displacement in children with cerebral palsy. J Pediatr Orthop 35: 511-515.

19. Mutlu C, Cullu E, Omurlu I (2018) The effect of hip reconstruction on gross motor function levels in children with cerebral palsy. Acta Orthop Traumatol Turc 52: 44-48.

20. Barakat MJ, While T, Pyman J, Gargan M, Monsell F, et al. (2007) Bilatera hip reconstruction in severe whole-body cerebral palsy: ten-year follow-up results. J Bone Joint Surg Br 89: 1363-1368

21. Root L, Laplaza FJ, Brourman SN, Angel DH (1995) The severely unstable hip in cerebral palsy. Treatment with open reduction, pelvic osteotomy, and femoral osteotomy with shortening. J Bone Joint Surg Am 77: 703-712.

22. Terjesen $T$ (2017) To what extent can soft-tissue releases improve hip displacement in cerebral palsy? Acta Orthop 88: 695-700. 